\title{
Application of LDA to bubbly flows
}

\author{
R.F. Mudde *, J.S. Groen, H.E.A. Van Den Akker \\ Kramers Laboratorium voor Fysische Technologie, Department of Applied Physics, Delft University of Technology, \\ Prins Bernhardlaun 6, 2628 BW Delft, The Netherlands
}

Received 23 February 1998; accepted 23 February 1998

\begin{abstract}
The fluctuating velocity field in an air-water bubble column (i.d. $15.2 \mathrm{~cm}$ ) at a gas fraction of $25 \%$ is investigated using backscatter LDA. Since the interpretation of LDA signals in bubbly flows is not straight forward also experiments on a single bubble train are reported. It is discussed that in the latter case when using seeding the backscatter LDA measures predominantly the liquid velocity. No improvement from thresholding on the discrimination between gas and liquid was found. The bubble column experiments show that the radial averaged liquid velocity profile represents the well known gross scale circulation present in the column. More interesting, it is also seen that the fluctuating velocity field can be studied in great detail. The velocity probability density functions directly indicate high turbulence intensity. Low frequency fluctuations are observed in agreement with visual observations. The data rate is an exponential function of the distance from the column wall. This limits the possibilities of spectral analysis in the central part of the flow. However, close to the wall the mean data rate is sufficient to study the frequency contents of the signal. It is shown that the power spectral density function obeys a $-5 / 3$ power law and that the autocorrelation function is of similar shape as reported in literature on bubbly flows. (c) 1998 Elsevier Science B.V. All rights reserved.
\end{abstract}

\section{Introduction}

Bubbly flows are frequently encountered in industrial applications ranging from biotechnology to chemical engineering or the nuclear industry. Nowadays, the problems associated with these applications (like scale up) are treated experimentally, theoretically and numerically. Especially the use of computational fluid dynamics (CFD) has a lot to offer to the field of dispersed multiphase flows. However, the use of CFD is still hampered

\footnotetext{
* Corresponding author. Tel.: + 3115 2781400/2834; fax: +31 15 2782838; e-mail: rob@klft.tn.tudelft.nl
}

by a lack of understanding of the basic flow phenomena. Furthermore, validation of the simulated results is almost always required. Both aspects call for detailed experiments on the flow properties. Reports on quantification of the local, rather than the global hydrodynamic flow phenomena are scarce in literature, especially if the fraction of the dispersed phase increases. One of the reasons is, obviously, the difficulties encountered in experiments on multiphase flows. The flow is difficult to access and very sensitive to disturbances caused by, e.g. intrusive measuring probes. Therefore, the use of non-intrusive optical techniques (like PIV and LDA) could be benefi-

0029-5493/98/\$ - see front matter (C) 1998 Elsevier Science B.V. All rights reserved.

PII S0029-5493(98)00206-4 
cial. This paper addresses the possibilities of LDA in bubbly flows, i.e. air-water bubble columns, at moderate gas fraction. The target is to measure the liquid velocity and subtract relevant information concerning 'vortical structures' and turbulent quantities from the measurements. LDA, however, is less straight forward in bubbly flows than in single phase flows.

\section{Bubble columns}

The bubble column is one of the simplest devices in which dispersed two phase flow occurs. Yet, its flow properties are still not understood to a level that makes scale-up trivial. Nowadays, it has become apparent that studying the instantaneous flow field is the key to understanding the bubble column. A few experimental techniques have the potential of providing the required data in a non-intrusive way: PIV (Chen et al., 1994), CARPT (Devanathan et al., 1990) and LDA, each with its limitations.

The flow in a bubble column is generated by the homogeneous aeration with gas through the bottom plate of the column. Due to density differences between a gas bubble and liquid, but also on a coarser scale between different regions in the mixture, a fluctuating flow field is observed. A long-time average of this field yields a gross-scale circulation of the liquid, with up flow in the center and down flow close to the wall (Hills, 1974). This is a consequence of the lower gas fraction close to the wall in comparison with the gas fraction in the central part of the column. This circulation is steady, but represents only a part of the flow phenomena. LDA can, in principle, be used to study the transient flow relatively close to the wall. However, an important question concerning LDA and bubbly flow has to be addressed: what is exactly measured by the LDA? Or stated differently: how does one discriminate between the motion of the gaseous bubble phase and the liquid? In this paper this question is addressed and some experimental findings in a bubble column (inner diameter $15.2 \mathrm{~cm}$ ) at a gas fraction of $25 \%$ (volume based) are reported.

\section{LDA}

In the literature various reports are found on the issue of discriminating between the different phases. Sheng and Irons (1991) have grouped these into three different main categories:

- discrimination based on the form of the bursts (Boerner et al., 1984);

- signal analysis and light blocking (Ohba et al., 1976; Lee and Srinivasan, 1982; Lee and Cho, 1984);

- difference in velocity distribution (Marié and Lance, 1983).

For the flow in a bubble column the third option can not be used since the fluctuations in the liquid velocity cause the liquid velocity probability density function (pdf) to overlap the velocity pdf of the gas bubbles. Forward scatter, or more general usage of one of the laser beams that travels through the two phase flow, is not possible since the interest is in moderate gas fractions (up to $25 \%$ ) in bubble columns with diameters of $15 \mathrm{~cm}$ and higher. As a consequence light blocking techniques can not be used. As a result, the experiments are restricted to backscatter.

Velidandla et al. (1996) reported experiments in a bubbly flow with backscatter LDA. They found that the bursts of the seeding particles could be separated well from those of the bubbles. One reason is in the difference in liquid and gas velocities present in their experiments (and therefore different frequency shifts used when studying liquid or bubbles). For those bubble bursts that pass this first frequency 'filter' a threshold on the pedestal amplitude was used to remove them. In this way Velidandla et al. were able to remove most of the measured bubble velocities. However, this procedure can not be used when dealing with bubble columns, since the velocities of the gas and the liquid are of the same order, moreover even the bubble velocity can be negative.

To be able to use the LDA in bubble columns with sufficient confidence a single bubble train experiment similar to the one of Sheng and Irons (1991) was set up. The objective of this experiment is to study in detail the velocity field around a single bubble with forward scatter and compare the findings with the same experiments but measured with backscatter. 

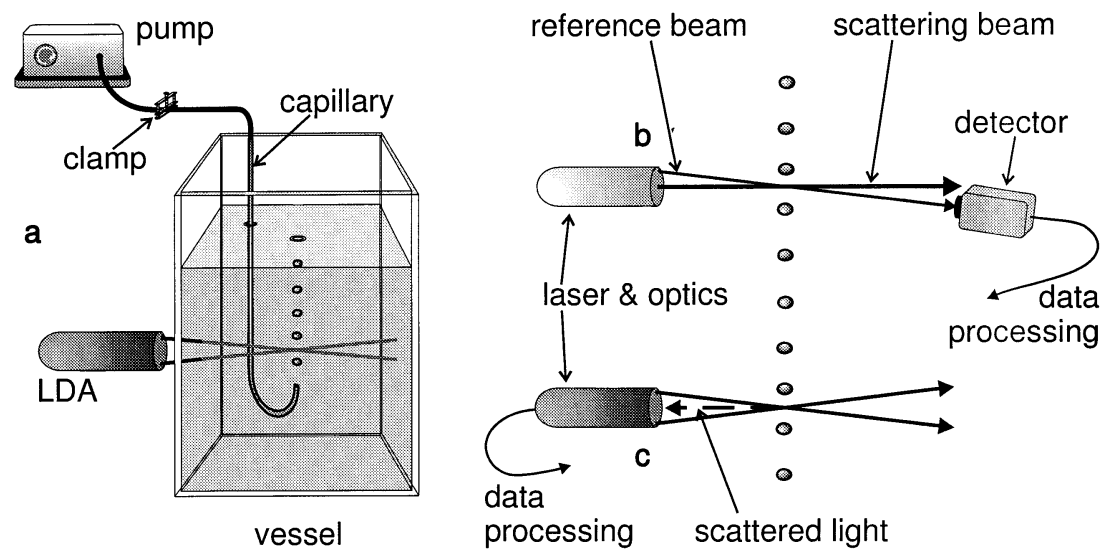

Fig. 1. Set up.

\section{Set up}

In Fig. 1 the set up is shown. It consists of a large rectangular vessel filled with water. A single capillary (1 $\mathrm{mm}$ i.d.) is inserted into the liquid and air is pumped through it at an adjustable rate. In this way a single bubble train is formed, with ellipsoidal, wobbling bubbles (vertical size $\sim 2.5$ $\mathrm{mm}$, horizontal size $\sim 4 \mathrm{~mm}$ ) at a bubble frequency ranging from 6 to $28 \mathrm{~Hz}$. The Reynolds number of these bubbles is $\sim 750$, which means that their motion is zigzag instead of rectilinear and that a wake behind the bubbles is present. The terminal velocity of these bubbles is $25 \mathrm{~cm}$ $\mathrm{s}^{-1}$ (from time of flight measurements). The forward scatter LDA equipment consists of a $15 \mathrm{~mW}$ Spectra-Physics $\mathrm{HeNe}$ laser, a rotating grating (preshift $827 \mathrm{kHz}$ ), a $400 \mathrm{~mm}$ lens (fringe spacing: $12.2 \mu \mathrm{m}$, measuring volume $4 \times 0.2 \mathrm{~mm}$ ). A photodiode detects the scattered light and the reference beam. In the backscatter experiments a $4 \mathrm{~W}$ Spectra-Physics $\mathrm{Ar}^{+}$laser with a TSI 9201 colorburst beam separator is used. The frequency preshift is $1 \mathrm{Mhz}$. The beam pair is focussed with a $350 \mathrm{~mm}$ lens (fringe spacing $3.61 \mu \mathrm{m}$, measuring volume $0.8 \times 0.1 \mathrm{~mm}$ ). The detected light is sent into a TSI 9230 colorlink. The detected bursts are processed with processor types IFA 550 and IFA 750 , controlled by a $486 / 66 \mathrm{MHz}$ PC. Experiments are performed $2.5 \mathrm{~cm}$ above the outlet of the capillary; only vertical velocities are registered.

\subsection{Forward scatter}

In the forward scatter experiments a high data rate was obtained $(\sim 30 \mathrm{kHz})$. The voltage supply of the photomultiplier is set at a relatively low value $700 \mathrm{~V}$ so that noise influences are minimized. In Fig. 2 the pdfs of the measured velocities are shown for three different positions in the bubble train: (a) at the center line (relative to the capillary), (b) at the edge (i.e. $2 \mathrm{~mm}$ from the center line) and (c) outside the train (i.e. $2 \mathrm{~cm}$ from the center line). Especially Fig. 2a seems to suggest that both the bubble and liquid velocity are registered. The pdf consists of a large slightly off-zero peak, showing that the liquid is entrained by the bubbles and a small peak at velocities $\sim 25$ $\mathrm{cm} \mathrm{s}^{-1}$. The latter value equals the sum of the rise velocity of a single bubble and the mean of the entrained liquid velocity. Similar findings have been reported by Marié and Lance (1983). They found that for liquid velocities above $20 \mathrm{~cm} \mathrm{~s}^{-1}$ the pdf shows the two peaks: a large one centered around the liquid velocity and a small one at higher velocities. The latter was attributed to the velocity of the bubbles.

As was already pointed out by Sheng and Irons (1991), a bubble present at the measuring volume blocks the reference beam and the photo multiplier is temporarily blinded. This is most clearly seen by zooming in on a time series of the velocity when one bubble is passing (see Fig. 3). When a 

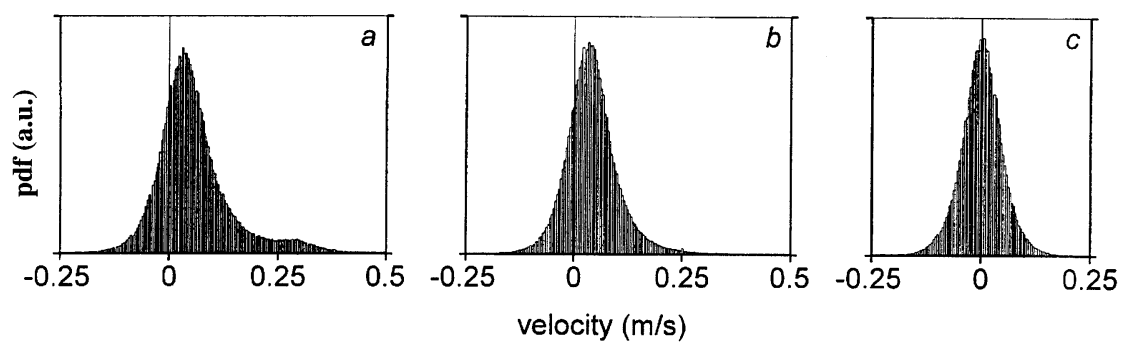

Fig. 2. pdfs at different positions: (a) at center line, (b) at edge of bubble train, (c) outside bubble train.

bubble approaches the focus, an increase in velocity is seen. This is still liquid velocity. As a consequence of the zigzag motion the magnitude of the velocity may exceed the steady terminal velocity of the bubble. The wobbling interface of the bubble may in principle generate a burst (Durst and Zare, 1975), but the data rate of these events is too low to account for the small peak $\sim 25 \mathrm{~cm} \mathrm{~s}^{-1}$ in the pdf. A similar conclusion has been reported by Ohba et al. (1986) by using a forward and a sidescatter set up at the same time.

When the bubble passes the measuring volume no bursts are detected, so a gap in the signal is created. Note that indeed the duration of this gap is, roughly speaking, equal to the ratio of the vertical size of the bubble and the bubble rise velocity. After the bubble has passed through the focus, again 'high' velocities are measured. This time the wake of the bubbles is detected. The size of the wake is seen to be similar to the bubble size. Since the Reynolds number of the bubbles is $\sim 750$, a well developed wake is present with velocities that may be higher than the bubble velocity.

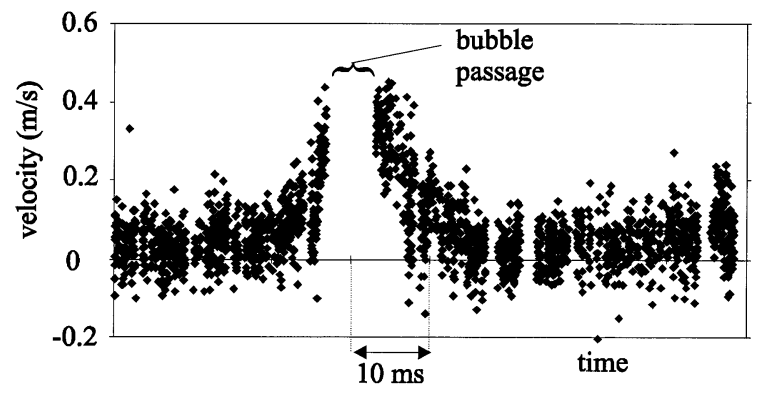

Fig. 3. Passage of a single bubble.
Durst et al. (1984) observed that the interface of bubbles with a diameter of $6 \mathrm{~mm}$ rising in a viscous liquid (Reynolds number 0.14) contributed to the velocity measurements only in case the measurements take place exactly at the center line of the bubble motion. In all other cases the light is not scattered in the direction of the photomultiplier. Martin et al. (1981) investigated LDA signals for forward scatter with small air bubbles (dia $0.2-1.0 \mathrm{~mm}$ ). They found Doppler bursts with three peaks which could be connected to the position of the bubble with respect to the measuring volume. No triple peaked burst were found in the present analysis, most likely due to the larger bubble with its deforming interface.

\subsection{Back scatter}

In the back scatter mode the data rate is much lower. The data rate without addition of seeding is $10-25 \mathrm{~Hz}$. The addition of seeding (alumina coated spherical polyethylene particles of $4 \mu \mathrm{m}$ diameter, density $2.6 \cdot 10^{3} \mathrm{~kg} \mathrm{~m}^{-3}$ ) increases this rate to some $1000 \mathrm{~Hz}$. No seeding particles were found on the bubble-liquid interface. This difference in data rate indicates that in the backscatter mode predominantly the liquid velocity is registered. This can also be seen by looking at the pdfs of forward and backscatter experiments. An example is shown in Fig. 4 for measurements at the center line of the bubble train at a bubble frequency of $6 \mathrm{~Hz}$. The shape of the pdfs is similar. Both have a big and small peak at the same velocity. The width of the backscatter pdf is, however, considerably smaller. This is caused by the much smaller measuring volume in the backscatter case and to a less extent to a more 

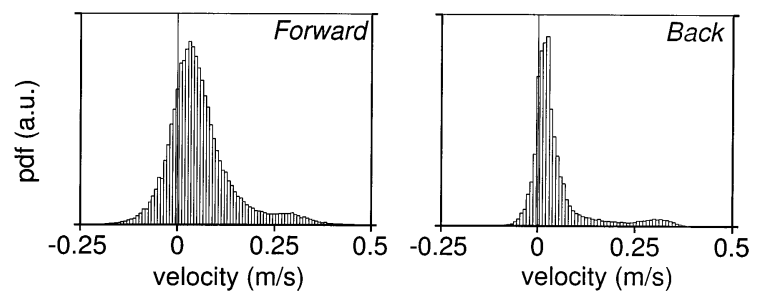

Fig. 4. Comparison between forward scatter and backscatter.

constant frequency preshift. In Fig. 5 a comparison is made between a time series in forward and backscatter. Although in backscatter the data rate is much too low to allow for a detailed analysis of the flow when a bubble passes through the measuring volume, still the wake and the approach of individual bubbles can be seen.

A further test is applied by thresholding the burst amplitude. This is done for the three measuring positions mentioned above. Fig. 6a shows the data rate normalized by the rate with a threshold value of $20 \mathrm{mV}$. It is seen that the decrease in data rate is independent of whether or not the measurements were performed in or outside the bubble train. This again seems to indicate that predominately liquid velocities are registered. Increasing the voltage supply to the photo multiplier resulted in more 'bursts' above the threshold value, but those bursts were usually not validated by the IFA.

Finally, the measured average velocity is shown as a function of the threshold value in Fig. 6b. Here a slight increase in the averaged velocity in

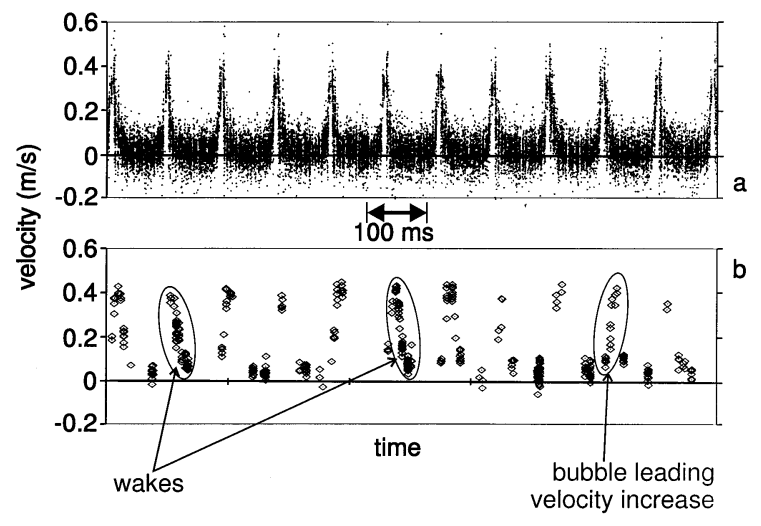

Fig. 5. Time series for (a) forward and (b) backscatter. the train is found, showing that thresholding favors detection of 'high' velocities. Even at the highest threshold value the averaged velocity is still much lower than the bubble velocity. From the above we have concluded that in the backscatter mode, the LDA predominantly measures the velocity of the liquid and that phase discrimination based on burst amplitude offers no significant improvement.

\section{Bubble column experiments}

The experiments in the bubble columns have been performed all with the backscatter configuration and the IFA 750 processors. The column (inner diameter $15.2 \mathrm{~cm}$ ) is equipped with a porous plate distributor at the bottom (pore diameter $40 \mu \mathrm{m}$ ). The liquid phase (tap water) is in batch, and the gassed height of the mixture is kept constant at a value of 8 diameters in all cases. The air, introduced via the porous plate, forms bubbles with a volume equivalent diameter of $3 \mathrm{~mm}$. The superficial gas velocity ranges up to $7 \mathrm{~cm} \mathrm{~s}^{-1}$, corresponding to a gas fraction of more than $30 \%$. Due to high turbulence intensities and dirty water hardly any larger bubbles are detected at these high gas fractions (measured with a 4-point glass fiber probe (Frijlink, 1987)).

In all experiments the flow was seeded with the particles mentioned above. The maximum data rate was $1000-1400 \mathrm{~Hz}$ when measuring close to the wall. In Fig. 7 a typical radial profile of the averaged axial liquid velocity is shown (at $39 \mathrm{~cm}$ above the gas distributor). Clearly a gross scale circulation is observed. This has been reported in numerous papers, with a variety of measuring techniques such as Pitot tubes, hot wire anemometers, radioactive tracers particles, PIV, etc. This averaged axial velocity profile is symmetric and it is found that indeed there is no net liquid flow through a horizontal cross section of the column. More interesting is, however, the development of the axial velocity pdfs (also shown in Fig. 7). As is seen the fluctuations around the means are large everywhere in the column. This is the general picture found for all gas fractions investigated. Even in the center of the column the 

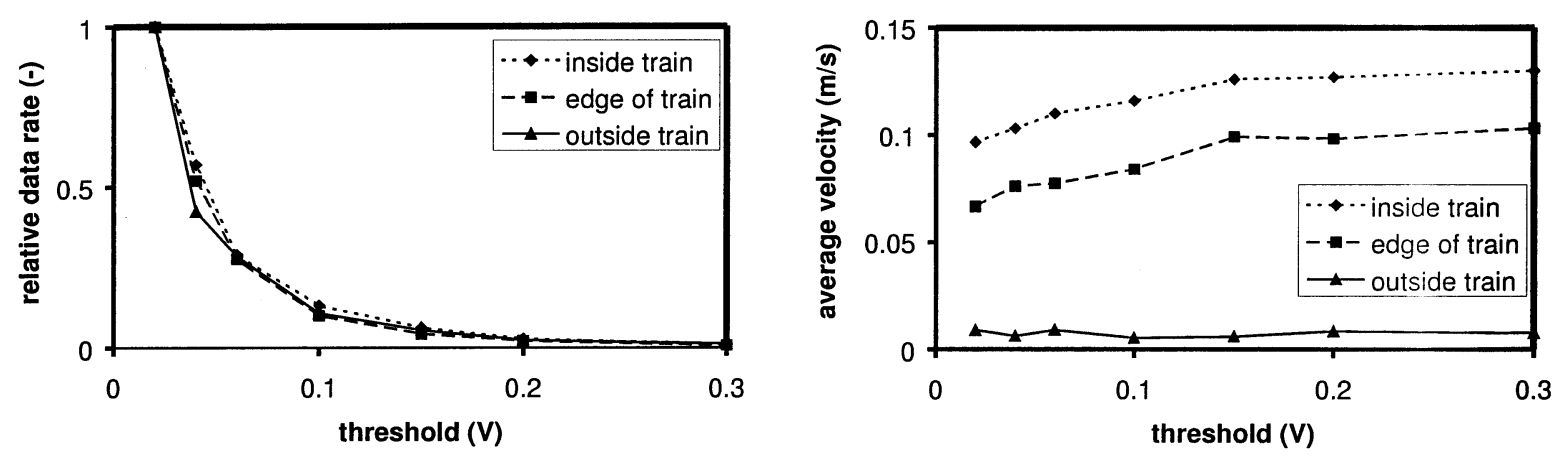

Fig. 6. Effect of thresholding.

liquid is found to flow downward every now and then. Analysis of the pdfs shows that turbulence intensities, defined as the ratio the root-meansquare (rms) value of the fluctuating part of the velocity and the mean of the absolute value of the axial velocity, are on the order of $100 \%$ or more. In Fig. 7 also a part of the radial profile of the rms of the axial velocity is shown. The dip around $r / R=0.8$ is found for other gas fractions as well. No data are presented for $r / R<0.4$ because the number of data points per position in that part is insufficient for a reliable estimate of the rms. Duplication of the measurement shows that close to the wall the uncertainty in the means is less than $5 \%$. But when probing deeper into the column this uncertainty increases. The latter is caused by the decreased number of data that is available. It is expected that this uncertainty can be greatly reduced by measuring much longer.

The data rate is a strong function of the distance from the wall. Obviously, this is caused by the decreasing probability that the path of the lasers beams is open due to the presence of the bubbles. Ohba et al. (1976) have shown that the probability of a laser beam to penetrate into a bubbly flow decreases exponentially. Or in terms of the ratio between the received intensity with bubbles $I$ and without bubbles $I_{0}$ (assuming spherical bubbles):

$\frac{I}{I_{0}}=\exp \left(-\frac{3}{2} \frac{l}{d_{\mathrm{b}}} \alpha\right)$

with $l$ the path length through the two phase mixture, $d_{\mathrm{b}}$ the bubble diameter and $\alpha$ the gas fraction (for ellipsoidal bubbles the long horizontal axis has to be used).

When measuring with LDA the two beams have to form the measuring volume. Thus, both paths for the beams have to be 'open' simultaneously. Only if the distance between the beams is sufficiently large will these occur independently from one another. It is expected that the data rate will follow the same dependence on distance in the bubbly flow as given by Ohba. This is indeed the case as is illustrated in Fig. 8. This plot shows the drop in data rate with increasing distance from the wall for operation at a gas fraction of $25 \%$. The constant $3 / 2$ from Eq. (1) has now to be replaced by 2.4 . This shows that indeed the data rate drops faster than a single beam is attenuated, but $2 \times 3 / 2$ for independent blockage of the beams is not found. Note that although the data rate in the central part of the column is very small it is still possible to obtain a reliable pdf and mean velocity (specially the latter can be checked to some extent by calculating the net mean axial flow in a horizontal cross section).

A better insight in the fluctuating nature of the flow can be obtained by analyzing a time series of the axial velocity. In Fig. 9a part of a series of 222 s (i.e. 297640 measuring points at mean data rate $1342 \mathrm{~Hz}$ ) is shown, while Fig. 9b zooms in on a small time interval. The series is taken at a value of 0.90 of the dimensionless radial position. The velocity data are sampled at irregularly spaced time intervals as in single phase flow, but due to blocking of bubbles large gaps are found in the time series. When a moving average is applied, the 


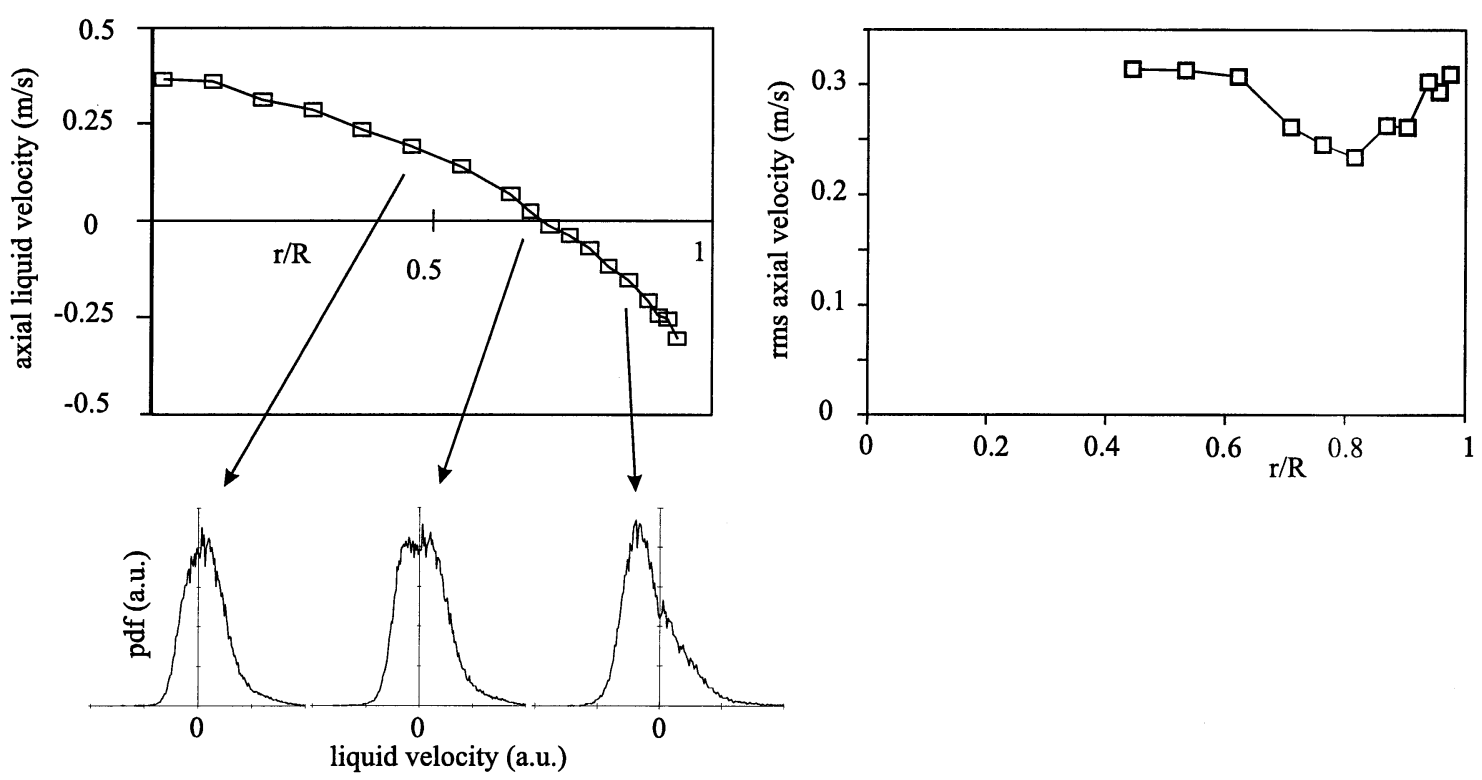

Fig. 7. Radial profile of the axial liquid velocity and its rms for a gas fraction of $25 \%$.

presence of low frequency fluctuations $(\sim 0.1 \mathrm{~Hz})$ in the liquid velocity is revealed. These are caused by large 'vortical structures' that are observed in the flow (Groen et al., 1996).

Calculation of the auto power spectral density of the time series from LDA measurements is complicated by the uneven distributions of the samples in time. Adrian and Yao (1987) investigated (for single phase flow) the consequences of a sample and hold for resampling the Poisson

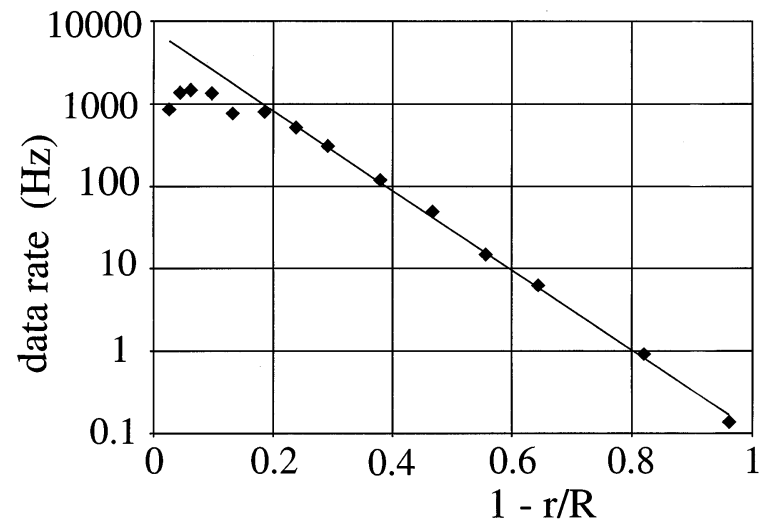

Fig. 8. Data rate versus dimensionless distance from the wall, gas fraction $=25 \%$. distributed sample times at the mean data rate. They showed that this results in low pass filtering the data with low pass filter frequency equal to the mean data rate divided by $2 \pi$ causing a $1 / f^{2}$ decay at high frequencies. Banning (1996) tested sample and hold and linear interpolation and
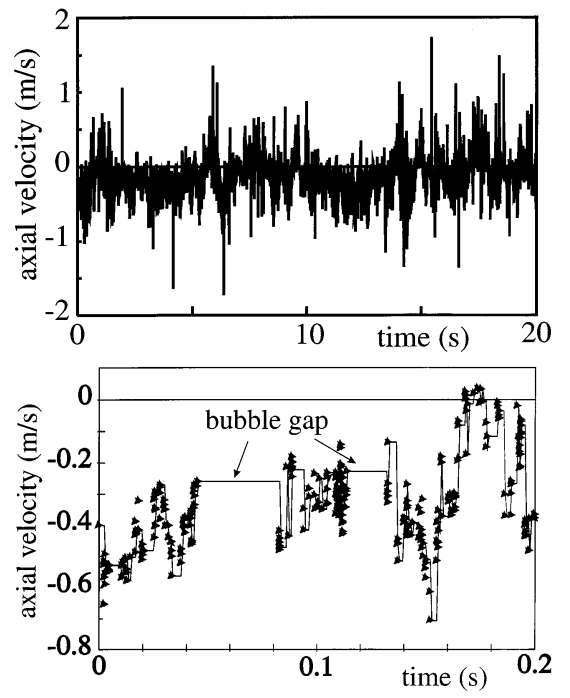

Fig. 9. Time series of axial velocity at a dimensionless radial coordinate of 0.90 . 

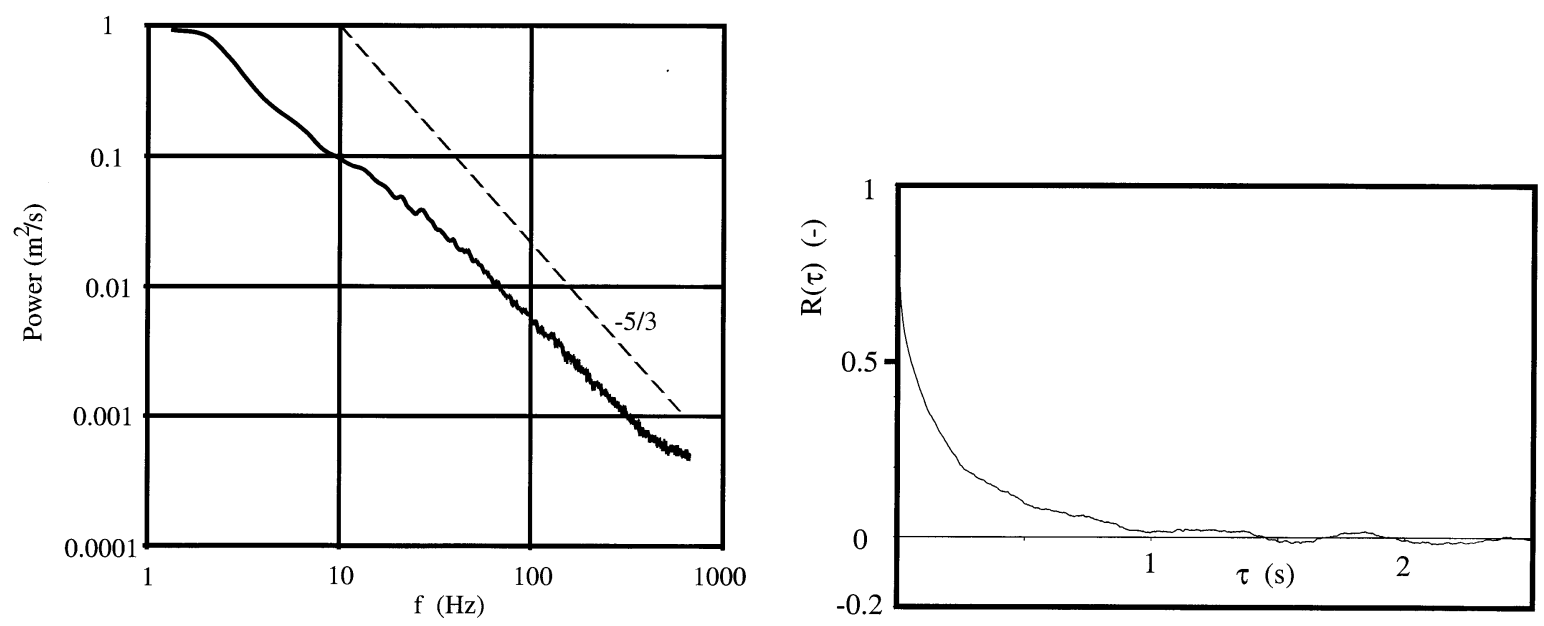

Fig. 10. (a) Power spectral density, (b) autocorrelation of the fluctuating velocity.

concluded that the latter leads to a less distorted estimate of the spectral density. In the case of the bubbly flow the resampling also has to close the blocking gaps. We have tested sample and hold as well as linear interpolation. It was found that both schemes give the same spectra. As an example the spectral density of the series from Fig. 9 is shown in Fig. 10a (obtained via sample and hold, the series is divided into 580 records of 1024 points). The levelling off at high frequencies is similar to the one reported by Banning (1996) and is a consequence of the resampling. Note that the spectrum seems to follow a $--5 / 3$ power law as found in many single phase flows. This differs from the $-8 / 3$ dependence reported by Lance and Bataille (1983) in low gas fraction cocurrent water air bubbly flow with grid generated turbulence. Note also that a rough estimate of the Kolmogorov time scale for the flow in the bubble column is $1.2 \mathrm{~ms}$, hence a frequency of $800 \mathrm{~Hz}$. When similar data series are recorded deeper into the column the data rate will drop as discussed. Spectral densities calculated according to the same procedure have the same form but are shifted towards lower frequencies. The $f^{-2}$ decay due to the sample and hold procedure as discussed by Adrian and Yao (1987) is not observed. This may be caused by the much higher data rate in between the bubble gaps, so that the effect of filtering sets in at a much higher frequency than the overall mean data rate divided by $2 \pi$. The distortion of the spectra by the resampling is still under investigation.

From the spectral density the autocorrelation is calculated. The data series from Fig. 9 is now divided into records of 24.4 s (i.e. 32768 points). This is the minimal length that has to be used to capture the low frequency vortical structures. The result is shown in Fig. 10b. It clearly shows that this flow is different from single phase turbulence (Nieuwstadt, 1992). The shape of the autocorrelation is the same as reported by Lübbert (1983), who measured the axial velocity in a bubble column using a hot wire and by Lance and Bataille (1983) for bubbly flow with grid generated turbulence. The latter reported this particular shape for the autocorrelation already at gas fractions of $0.5 \%$. From the autocorrelation the integral time scale is calculated according to (Devanathan 1991)

$T_{I}=\frac{\int_{0}^{\infty} t^{\prime}\left|R\left(t^{\prime}\right)\right| \mathrm{d} t^{\prime}}{\int_{0}^{\infty}\left|R\left(t^{\prime}\right)\right| \mathrm{d} t^{\prime}}$

with $R(t)$ the autocorrelation function. The integral time scale is $0.43 \mathrm{~s}$ which is similar to the findings of Devanathan (1991) who obtained integral time scales on the order of $0.5 \mathrm{~s}$ from tracking a neutrally buoyant radioactive particle in bubble columns. 


\section{Concluding remarks}

The flow in a bubble column at moderate gas fraction is investigated using LDA. Due to the relatively high gas fraction forward scatter LDA cannot be used. Moreover, various techniques to discriminate between data from the gas or liquid phase cannot be used since the velocity distributions of both phases show a considerable overlap. Therefore, a comparison between forward scatter and backscatter LDA in a single bubble train is made. It shows that in backscatter predominantly the liquid velocity is measured. Applying thresholds to try to eliminate contributions from the bubbles did not have a significant effect on the data registered inside or outside the bubble train. The above strongly suggest that in backscatter (with proper seeding) the liquid velocity is measured.

The experiments in the bubble column (at a gas fraction of $25 \%$ ) shows that it is possible to obtain relevant time series even relatively deep into the flow. The mean data rate, however, drops exponentially with the distance from the wall, in agreement with theoretical considerations. Spectral analysis of the time series seems to indicate that: (i) the spectrum shows a $-5 / 3$ power law for the higher frequencies, (ii) the resampling with a sample and hold scheme does not introduce a $1 / f^{2}$ decay and (iii) the autocorrelation function is different from the single phase flow case but in agreement with literature findings. The obtained integral time scale is similar to that reported by Devanathan et al. (1990).

Further research on LDA and bubbly flows is still required to better understand the consequences of the 'big' bubble gaps in the signal. Especially, the distortion of the calculated spectra needs further attention.

\section{References}

Adrian, R.J., Yao, C.S., 1987. Power spectra of fluid velocities measured by laser Doppler velocimetry. Exp. Fluids 5, $17-28$.

Banning, R., 1996. On the spectral analysis of poisson sampled velocity measurements in turbulent flows. In: Proceedings of the IEEE Instrumentation and Measurement Technology Conference and the IMEKO Technical Committee 7, vol. 1, pp. 762-767.

Boerner, T., Martin, W.W., Leutheusser, H.J., 1984. Comparative measurements in bubbly two-phase flow using Laser Doppler and hot-film anemometry. Chem. Eng. Commun. 28, 29-43.

Chen, R.C., Reese, J., Fan, L.-S., 1994. Flow structure in a three-dimensional bubble column and three-phase fluidized bed. AIChE J. 49 (7), 1093-1104.

Devanathan, N., 1991. Investigation of liquid hydrodynamics in bubble columns via a computer automated radioactive particle tracking (CARPT) facility. PhD thesis, Washington University, Saint Louis, Missouri.

Devanathan, N., Moslemian, D., Dudukovic, M.P., 1990 Flow mapping in bubble columns using CARPT. Chem. Eng. Sci. 45 (8), 2285-2291.

Durst, F., Zare, M., 1975. Laser-Doppler measurements in two-phase flows. In: Proceedings of the LDA-Symposium. Copenhagen, Denmark, pp. 403-429.

Durst, F., Taylor, A.M.K.P., Whitelaw, J.H., 1984. Experimental and numerical investigation of bubble-driven laminar flow in an axisymmetric vessel. Int. J. Multiph. Flow 10 (5), 557-569.

Frijlink, J.J., 1987. Physical aspects of gassed suspension reactors. PhD thesis, Delft University of Technology, The Netherlands.

Groen, J.S., Oldeman, R.G.C., Mudde, R.F., Van Den Akker, H.E.A., 1996. Coherent structures and axial dispersion in bubble column reactors, Chem. Eng. Sci. 51 (10), $2511-$ 2520.

Hills, J.H., 1974. Radial non-uniformity of velocity and voidage in a bubble column. Trans. I. Chem. Eng. 52, 1-9.

Lance, M., Bataille, J., 1983. Turbulence in the liquid phase of a bubbly air-water flow. In: Kakaç, S., Ishii, M. (Eds.), Advances in Two-Phase Flow and Heat Transfer. Nijhoff, Boston, pp. 403-427.

Lee, S.L., Cho, S.K., 1984. Simultaneous measurement of size and two-velocity components of large droplets in a twophase flow by Laser Doppler anemometry. In: Proceedings of the Symposium Measuring Techniques in Gas-Liquid Two-Phase Flows. Nancy, France, pp. 149-164.

Lee, S.L., Srinivasan, J., 1982. An LDA technique for in situ simultaneous velocity and size measurement of large spherical particles in a two-phase suspension flow. Int. J. Multiph. Flow 8 (1), 47-57.

Lübbert, A., 1983. Turbulence measurements in bubble columns. In: Alper, E. (Ed.), Mass Transfer with Chemical Reaction in Multiphase Systems: Two-Phase Systems, vol. 1:. Nijhoff, The Hague, pp. 553-564.

Marié, J.L. Lance, M., 1983. Turbulence measurements in two-phase bubbly flows using Laser Doppler anemometry. In: Proceedings of the Symposium on Measuring Techniques in Gas-Liquid Two-Phase Flows. Nancy, France, pp. $141-148$. 
Martin, W.W., Adbelmessih, A.H., Liska, J.J., Durst, F., 1981. Characteristics of Laser Doppler signals from bubbles. Int. J. Multiph. Flow 7, 439-460.

Nieuwstadt, F.T.M., 1992. Turbulentie. Epsilon Editions, Utrecht [in Dutch].

Ohba, K., Kishimoto, I., Ogasawara, M., 1976. Simultaneous measurement of local liquid velocity and void fraction in bubbly flows using a gas laser-part I: principle and measuring procedure. Technol. Rep. Osaka Univ. 26 (1328), 547-556.
Ohba, K., Yuhara, T., Matsuyama, H., 1986. Simultaneous measurements of bubble and liquid velocities in two-phase bubbly flow using laser Doppler velocimeter. Bull. JSME 29 (254), 2487-2493.

Sheng, Y.Y., Irons, G.A., 1991. A combined Laser Doppler anemometry and electrical probe diagnostic for bubble two-phase flow. Int. J. Multiph. Flow 17 (5), 585-598.

Velidandla, V., Putta, S., Roy, R.P., 1996. Velocity field in isothermal turbulent bubbly gas-liquid flow through a pipe. Exp. Fluids 21, 347-356. 